\title{
Por un mundo libre de tuberculosis
}

\author{
Dr. Juan Pineda Olvera* \\ Profesor Titular A de la Facultad de Estudios Superiores Iztacala UNAM
}

El 24 de marzo de cada año, se celebra una jornada a nivel mundial para formar conciencia en la población -a todos los niveles- sobre la amenaza que representa la tuberculosis (TB) como una enfermedad reemergente, devastadora y de dimensiones epidémicas, pues a pesar de los esfuerzos que se han emprendido desde hace años para su control, estos no han sido suficientes, pues se requiere de promoción y educación para la salud, de compromiso, recursos financieros y de la participación de todos los sectores sociales para su prevención, cura y control.

\section{ORIGEN DE ESTA CELEBRACIÓN}

En este año se cumplió el 127 aniversario del descubrimiento del bacilo que causa la TB, en virtud de que el 24 de marzo de 1882 el eminente investigador alemán Roberto Koch discípulo de Luis Pasteur, anunció el descubrimiento del agente biológico que trasmitía la enfermedad, acción que fue el paso más importante para el control de la misma. Vale la pena señalar que la inspiración de Roberto Koch podría adjudicársele a su esposa Frau Koch, quien le obsequió un microscopio en su cumpleaños; hecho que le permitió al científico, transformar todo un modelo de concepción de la enfermedad, transitando de la teoría de la generación espontánea a la unicausal para enfermedades de origen biológico. Este descubrimiento le llevó a Koch, a ganar en ese mismo año el Premio Nobel de Medicina.

Un siglo más tarde del descubrimiento del bacilo (1982), la Organización Mundial de la Salud (OMS) y la Unión Internacional contra la Tuberculosis y las Enferme- dades Respiratorias (UICTER) patrocinaron el primer día Mundial de Lucha contra la Tuberculosis con el fin de dar a conocer y de educar a la población en general sobre las graves consecuencias que acarrea el lastre de esta enfermedad en todas las economías, pero principalmente en las de países del Tercer Mundo y el repunte simultáneo de la enfermedad en las economías desarrolladas en las que influye definitivamente como un factor importante, la migración.

La TB hasta el descubrimiento de la estreptomicina, había sido considerada como la enfermedad más mortífera que hubiera aquejado al género humano. Hipócrates en el siglo V a.C. la definió como la enfermedad "más grave de todas, la de curación más difícil y la más fatal".

\section{ENTRE LA HISTORIA Y LA ACTUALIDAD}

Vale la pena señalar que la Tuberculosis es una enfermedad tan antigua como la especie humana, pues existen escritos y vestigios en momias de diferentes latitudes que dan indicios de su presencia y se hace hasta difícil explicar cómo en el ya entrado siglo XXI con los avances científicos y tecnológicos de punta (biología molecular, genoma humano y la nanotecnología; entre otros), no se hayan podido encontrar los mecanismos suficientes para el control y erradicación de la enfermedad, lo que hace develar el hecho de que no basta con la presencia del bacilo y de los deficientes mecanismos de respuesta inmunológica del sujeto, pues para conseguir el desarrollo de la misma, es necesaria la presencia e influencia de factores ambientales, económicos, políticos, antro- 
pológicos de la persona afectada, de su familia y de la sociedad, así como deficiencias en la infraestructura y en el funcionamiento de los servicios de salud y de los programas.

Cada año que los organismos internacionales de lucha contra la TB organizan un nuevo evento, las acciones se dirigen con la guía de una temática central, así por ejemplo en 1999, el Director de la Organización Panamericana de la Salud (OPS) Dr. George Alleyne, advirtió "estamos entrando en el siglo XXI con formas más peligrosas de la tuberculosis que amenazan nuestros países, aunque es una enfermedad curable y tenemos las herramientas para controlarla... En las Américas, hemos erradicado la poliomielitis y estamos en camino a la eliminación del sarampión, de manera que nos preocupa que los países todavía no estén asignando el mismo compromiso para el control de la tuberculosis. Nuestra tarea es poner el control de la TB como prioridad en la agenda". A 10 años de estas prerrogativas, el discurso del Dr.Alleyne, sigue vigente.

\section{LA TUBERCULOSIS POR EL MUNDO}

Algunos datos revelan que ara el 2006 existían 9.2 millones de casos nuevos (139 X 100,000), de los cuáles el 83\% correspondió a las Regiones de África, Asia Suroriental y el Pacífico Occidental, 14.4 millones de casos prevalentes, 4.1 (44\%) bacilíferos, 700,000 (8\%) VIH positivos, 500,000 multirresistentes y 1,7 millones de defunciones $(200,000$ con $\mathrm{VIH})$. Vale la pena señalar que entre 1980 y 2005 se notificaron más de 90 millones de casos.

La TB mata a los miembros productivos de la sociedad, cada segundo se infecta una persona por TB a nivel mundial, 3 de cada diez personas están infectadas y de 5 a 10\% de los infectados desarrollarán la enfermedad en algún momento de su vida. Además, un enfermo sin tratamiento infecta de 10 a 15 personas en un solo año, $50 \%$ de los enfermos morirán en 5 años si no reciben tratamiento y hay hasta un $50 \%$ de riesgo de desarrollar TB en personas con VIH positivo.

\section{LA TUBERCULOSIS EN MÉXICO}

Las estadísticas en el 2006 revelaron la existencia de 17,613 casos nuevos de TB en todas sus formas (84\% pulmonar, $1 \%$ meníngea, $5 \%$ ganglionar y $10 \%$ otras formas), 14,744 casos nuevos de TB pulmonar, 2116 defunciones de TB en todas sus formas, 515 casos fármacorresistentes acumulados, $20 \%$ de casos relacionados a diabetes mellitus, $5 \%$ de relación TB/SIDA y $10 \%$ de casos en Pediatría ( $<19$ años).

El Centro Nacional de Vigilancia Epidemiológica y Control de Enfermedades (CENAVECE, SSA), se señalan problemáticas relacionadas con la operación del Programa Nacional de Tuberculosis, como las deficiencias en la aplicación de la estrategia TAES, la atención heterogénea de casos fármacorresistentes (TB-MDR) y con comorbilidad por VIH, la falta de incorporación del sector privado y de hospitales de alta concentración a los procesos de atención, diagnóstico y tratamiento conforme a la normatividad oficial; además de insuficietes de acciones de promoción de la salud, participación comunitaria y comunicación de riesgos para adquirir la enfermedad y la limitada investigación operativa para tomar decisiones.

\section{Problemas internacionales en el control de la TB}

La Alianza STOP TB, señala como problemas en el control de la enfermedad:

1) Que no se está respondiendo a la tuberculosis farmacorresistente con la debida rapidez o compromiso. Hay que actuar ya, porque todos estamos en riesgo.

2) Líderes alrededor del mundo han reconocido la urgente necesidad de detener la coepidemia del VIH y la tuberculosis. Ahora, los países deben actuar ofreciendo servicios de tuberculosis a todas las personas que viven con $\mathrm{VIH}$ y servicios de $\mathrm{VIH}$ a todos los afectados por la tuberculosis.

3) Hay muchas interrogantes sobre la TB a las que no se puede responder mediante investigación científica. Necesitamos más investigación básica para entender mejor la compleja naturaleza de bacilo de la TB; nuevas y mejores medicinas, diagnósticos y vacunas; e investigación operativa para hacer más accesibles y eficientes los servicios de atención de este padecimiento.

4) Cuatro de cada diez personas que se enferman de tuberculosis no obtienen un diagnóstico y tratamiento correcto. Para llegar a ellos, se necesita que los programas públicos de TB se coordinen con otros actores que ofrecen servicios de tuberculosis, como proveedores privados y organizaciones religiosas.

\section{Tercer Foro de Socios de "Stop TB"}

Vale la pena destacar que para la celebración del Día Mundial de Lucha contra la TB de este año, se reunieron en Río de Janeiro, Brasil del 23 al 25 de marzo, más de mil 
especialistas y autoridades de todo el mundo en el Tercer Foro de Socios de "Stop TB" (Detengan la tuberculosis, en español), que es un movimiento global creado en el año 2000 para combatir la enfermedad y que agrupa a diferentes organismos multilaterales, gobiernos, organizaciones no gubernamentales, empresas y agencias de cooperación. La finalidad de la Reunión fue que a través de la OMS y del Banco Mundial, se intente comprometer a los gobiernos y a las empresas en el debate y en acciones concretas para disminuir la incidencia de esa afección a nivel mundial.

En este foro, la Dra. Margaret Chan, Directora General de la OMS, declaró "estos resultados ponen de manifiesto la urgente necesidad de detectar, prevenir y tratar la tuberculosis en las personas afectadas por el VIH, así como de someter a todos los enfermos de tuberculosis a pruebas del $\mathrm{VIH}$, con el fin de proporcionar prevención, tratamiento y atención. Para ello, los países necesitan programas de colaboración y sistemas de salud fortalecidos que luchen contra ambas enfermedades".
En su intervención, el Sr. Michel Sidibe, Director Ejecutivo del ONUSIDA afirmó: "tenemos que evitar que las personas afectadas por el VIH mueran de tuberculosis. El acceso universal a prevención, tratamiento, atención y apoyo en materia de VIH debe incluir la prevención, el diagnóstico y el tratamiento de la tuberculosis".

Dentro de la misma celebración que nos ocupa, la OMS publicó un informe denominado Informe 2009 sobre el control mundial de la tuberculosis, en el que señala que el número total de casos nuevos de tuberculosis se mantuvo estable en 2007 y el porcentaje de la población mundial que enfermó de tuberculosis continuó la lenta tendencia a la baja observada por primera vez en 2004. Reveló también que una de cada cuatro muertes por tuberculosis está relacionada con el $\mathrm{VIH}$, el doble de la proporción reconocida anteriormente. Se calcula que en 2007 hubo 1,37 millones de casos nuevos y 456000 muertes por tuberculosis en personas infectadas por el VIH. Esta cifra refleja una mejora en la calidad de los datos de los países afectados.

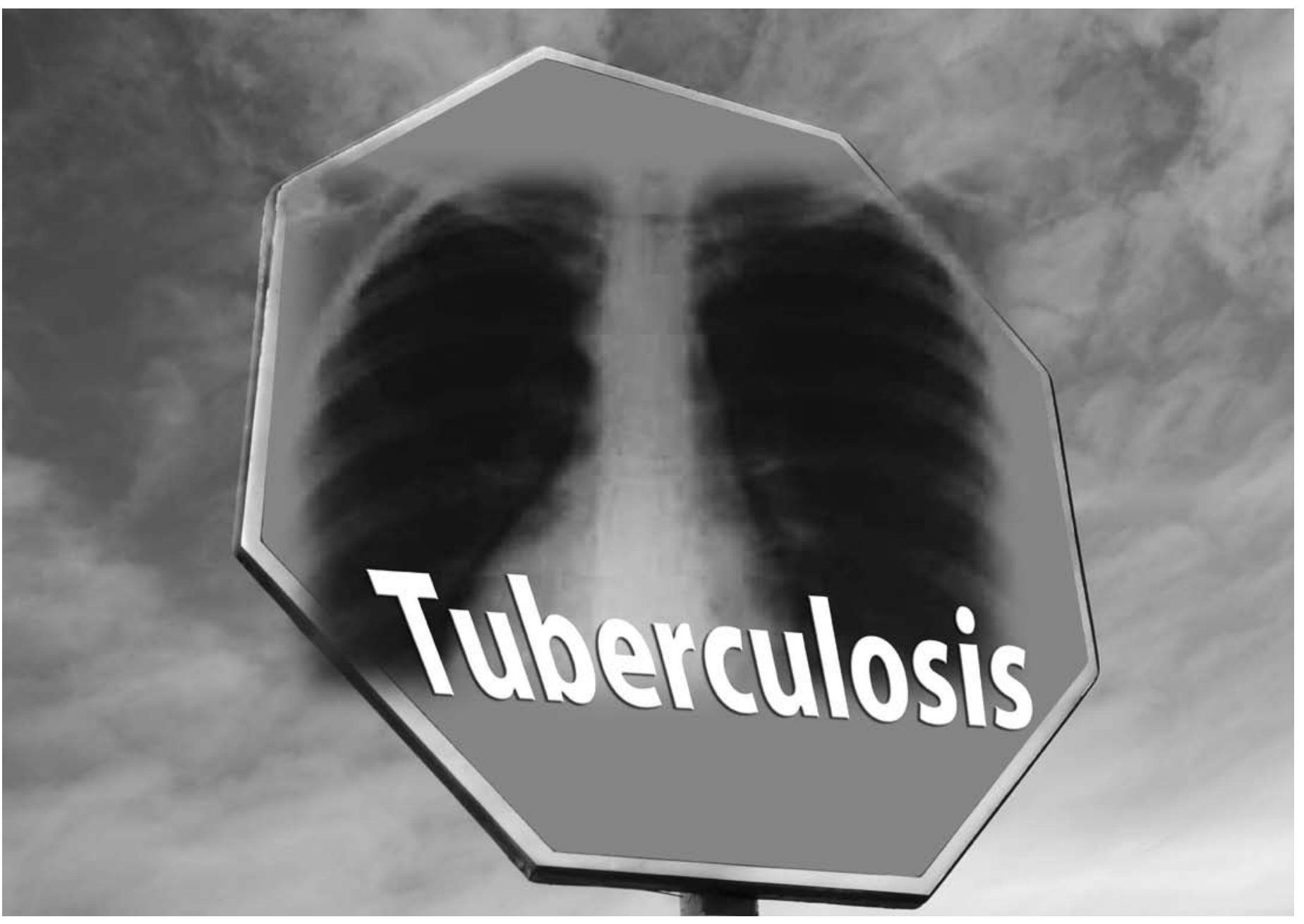




\section{Principales retos}

El informe en referencia señaló también que los principales retos que hoy enfrenta la sociedad en este rubro, son la coinfección con tuberculosis y VIH y las formas farmacorresistentes de tuberculosis. Se calcula que en 2007 unas 500000 personas padecían tuberculosis multirresistente, pero menos del $1 \%$ de ellas recibía tratamientos basados efectivamente, en las recomendaciones de la OMS.

El informe documenta respecto a la crisis económica mundial, la existencia de preocupaciones por una creciente escasez de financiamiento. El déficit de financiación para alcanzar los hitos de 2009 del Plan Mundial para Detener la Tuberculosis de la Alianza Alto a la Tuberculosis correspondiente a 94 países que integran este grupo, ha aumentado hasta alrededor de 1,5 miles de millones de dólares en EE.UU. Con una financiación plena, el Plan mundial logrará su objetivo de reducir a la mitad para el 2015 la prevalencia y mortalidad por tuberculosis con respecto a los niveles de 1990.

\section{Para finalizar}

Uno de los aspectos importantes que influirán para detener la TB es la formación de recursos humanos en salud, donde la enfermera y el enfermero representan un pilar a lo largo de tantos años de llevar la vacuna a los lugares más distantes, para la detección, seguimiento y control de casos y contactos. Entonces, merece especial reconocimiento el trabajo que nuestras escuelas y facultades de Enfermería, las que hacen su mejor esfuerzo en términos de incorporación de contenidos curriculares sobre la temática y el desarrollo de investigaciones operativas que involucren a docentes, estudiantes y prestadores de servicio social.

Finalmente manifiesto mi orgullo por el trabajo de nuestras colegas enfermeras de todo el mundo, quienes con su esfuerzo y trabajo cotidiano en las unidades de salud y en las comunidades, luchan incesantemente por combatir la tuberculosis y en casos frecuentes sin el apoyo de los gobiernos y de las instituciones, aun poniendo en riesgo su salud, su vida y su persona.

\section{“POR UN MUNDO LIBRE DE TUBERCULOSIS”}

\section{REFERENCIAS BIBLIOGRÁFICAS}

1 Alfonso, CR. Día Mundial contra la tuberculosis, 24 de marzo. Recuperado el 3 de marzo de 2009 de: http://edicionesanteriores.trabajadores.cu/SUPLEMENTO-SALUD/enfermedades-trasmisibles/tuberculosis.htm.

2 Ledermann-Dehnhardt, W. La tuberculosis antes del decubrimiento de Koch. Rev Chi Infect. Edición aniversario 2003; 46-473. Recuperado el 3 de marzo de 2009 de http://www.scielo.cl/scielo.php?pid=S0716-1018200302 0200015\&script $=$ sci_arttext

4 WHO report 2008. Global tuberculosis control: 2008 surveillance, planning, financing. Recuperado el 1 de abril de 2009, de http://data.unaids.org/pub/Report/2008/who2008globaltbreport_en.pdf

5 WHO. Stop TB Partnership-World TB Day 2009. Recuperado el 1 de abril de 2009 de: www.stoptb.org/events/world_tb_ day/2009/

6 MÉXICO. Secretaría de Salud (1993) - Modificación a la Norma Oficial Mexicana NOM-006-SSA2-1993, para la prevención y control de la tuberculosis en la atención primaria de salud.

7 Martha García-Avilez. MA. La tuberculosis más allá de las fronteras. Ponencia en la $3^{a}$. Jornada Universitaria de Lucha contra la tuberculosis Pulmonar. Marzo 17, 2009. FES Iztacala, UNAM.

8 Pineda-Olvera, J. 125 aniversario del descubrimiento del bacilo de Koch y 25 aniversario del Día Mundial de Lucha contra la Tuberculosis. Desarrollo Cientif Enferm. Vol. 15 No. 3 Abril 2007

\section{DIRECCIÓN PARA CORRESPONDENCIA}

Dr. Juan Pineda Olvera: juanpineda57@yahoo.com.mx 